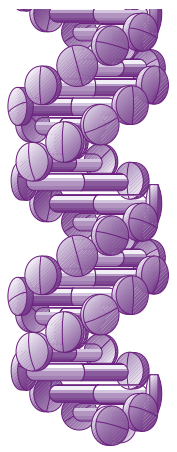

\title{
Donors FMO3 polymorphisms affect tacrolimus elimination in Chinese liver transplant patients
}

\begin{abstract}
Aim: Flavin-containing monooxygenase (FMO) variants were potentially involved in tacrolimus metabolism in kidney transplantion. The influences of FMO3 genotypes on tacrolimus elimination in Chinese liver transplant patients remained unclear. Patients \& methods: FMO3 SNPs and CYP3A5 rs776746 were analyzed in 110 Chinese patients. Results: Donor FMO3 rs1800822 allele T and rs909530 allele T were associated with fast tacrolimus elimination. Combination of polymorphisms of donor FMO3 rs1800822 and rs909530 genotype impacted on tacrolimus elimination $(p=0.0221)$. The number of donor rs 1800822 allele T and rs 909530 allele T was confirmed to be an independent predictor of the tacrolimus concentration-to-dose ratios for weeks 2,3 and 4 in the multivariate analysis. Conclusion: Donor's FMO3 polymorphisms might affect tacrolimus elimination.
\end{abstract}

First draft submitted: 2 June 2016; Accepted for publication: 17 July 2016; Published online: 13 January 2017

Keywords: CYP-450 3A5 $\bullet$ FMO3 $\bullet$ pharmacogenomics $\bullet$ tacrolimus

Tacrolimus is an immunosuppressant drug belonging to the calcineurin inhibitor class and is widely used in liver transplantation to prevent allograft rejection. It is characterized by a narrow therapeutic index and large interpatient variabilities in its pharmacokinetic and pharmacodynamic profiles. Therefore, tacrolimus-based immunosuppressive therapy can easily lead to either insufficient or excessive immunosuppression in liver transplant patients. Insufficient immunosuppression results in graft rejection while excessive immunosuppressive therapy can cause drug intoxication [1-3]. Such variability in drug response can result from genetic polymorphisms in drug-metabolizing enzymes, transport proteins and to a certain extent the target protein [4]. Pharmacogenetic studies, focusing on the effects of genetic polymorphisms on drug response, could provide useful clinical tools for tailoring immunosuppressive therapy to the genetics of an individual patient and their donor [5-7].

CYP3A5 is the main catalyst of tacrolimus. The genotype of rs776746 (CYP3A5 $6986 \mathrm{~A}>\mathrm{G}$ ) has a well-established influence on the pharmacokinetics of tacrolimus regardless of the population studied and is the best characterized pharmacogenetic marker of tacrolimus therapy. Almost all studies report that recipients with the $C Y P 3 A 5^{*} 3 /^{*} 3$ genotype (nonexpressers) exhibit higher dose-adjusted tacrolimus exposure and a lower dose requirement than in $C Y P 3 A 5^{*} 1{ }^{*} 1$ or ${ }^{*} 1 /{ }^{*} 3$ carriers (expressers) [8,9]. It is usually only possible to classify patients into one of these two categories; the proportion in the Han Chinese population is close to $50 \%$ [10]. However, residual interindividual differences in tacrolimus pharmacokinetics within the expressers and nonexpressers exist, demonstrating that different CYP3A5 genotypes cannot fully account for the drug response variations [11]. This is consistent with the fact that many drugs are subjected to multipathway metabolism. This has limited the clinical use of CYP3A5 rs776746 as a biomarker in individual immunosuppression therapy. In addition to $C Y P 3 A 5$ genetic variations, other genes involved in the metabolism and clearance pathway of tacrolimus may also be involved. It has been shown that CYP-450 $3 \mathrm{~A} 4$, the $A B C B 1$ gene that encodes the P-glycoprotein ATP-binding cassette transporter, the
Lei Ren ${ }^{\dagger, \neq, 1}$, Mujian Teng ${ }^{\ddagger \neq, 1}$, Tao Zhang $^{\dagger, \neq, 2}$, Xiaoqing Zhang ${ }^{3}$, Bo Sun ${ }^{4}$, Shengying Qin**,5, Lin Zhong ${ }^{2}$, Zhihai Peng ${ }^{2}$

\section{\& Junwei Fan ${ }^{* 2}$}

'Department of Hepatobiliary Pancreatic Surgery, Shandong Qianfoshan Hospital, Shandong University, Jinan 250014, China 2Department of Hepatobiliary Pancreatic Surgery, Shanghai First People's Hospital, School of Medicine, Shanghai Jiao Tong University, Shanghai 200080, China ${ }^{3}$ Department of Pharmacy, Shanghai Pulmonary Hospital, Tongji University School of Medicine, Shanghai 200433, China

${ }^{4}$ Department of Pharmacy, Shanghai First People's Hospital, School of Medicine, Shanghai Jiao Tong University, Shanghai 200080, China

${ }^{5}$ Bio-X Institutes, Key Laboratory for the Genetics of Developmental \& Neuropsychiatric Disorders (Ministry of Education), Shanghai Jiao Tong University, Shanghai 200030, China

*Author for correspondence:

fjwnet@163.com

**Author for correspondence:

chinsir@163.com

tFirst co-authors

${ }^{\ddagger}$ Authors contributed equally 
immunosuppressor IL-10 and the SLCO1B3 gene that encodes the organic anion-transporting polypeptide 1B3 may also contribute to interindividual differences intacrolimus disposition [12-15]. But their influence on the pharmacokinetics of tacrolimus appears limited. This has raised the question as to whether other genetic determinants influencing tacrolimus oxidative metabolism could help in dose individualization.

Flavin-containing monooxygenases (FMOs) represent the second most important group of human Phase I drug-metabolizing enzymes, after cytochrome P450. Several drugs are metabolized by both FMOs and CYPs. However, their role in drug metabolism has been underestimated compared with that of cytochrome P450 [16,17]. In humans, the FMOs' system includes five genes (FMO1-FMO5) and six pseudogenes (FMO6P-FMO11P). FMOs 1, 2, 3, 4 and 6P are located within a cluster spanning approximately $245 \mathrm{~kb}$, in the region q24.3. FMOs 7P-11P are located in the region q24.2. The FMO5 gene is closer to the centromere, in the region 1q21.1. To date, only FMOs 1,2 and 3 have been shown to play a significant role in drug metabolism [16,18]. There are striking tissuespecific differences in FMO gene expression. Expression of FMO1 is switched off in the liver shortly after birth and continues to be expressed in the adult kidney through the use of an alternative downstream promoter. Functional $\mathrm{FMO} 2$ protein is present in only a small percentage of the overall human population. FMO3 is expressed primarily in the liver after birth and is the major FMO isoform that contributes to hepatic drug metabolism [18,19]. The FMO3 gene is very polymorphic. FMO3 variants have also been shown to be associated to drug responses of sulindac (NSAID), ranitidine (histamine 2 receptor blockers), benzydamine (analgesic), amphetamine and methamphetamine (psycho-stimulant drugs) [17,20].

Little is known about the effect of $\mathrm{FMO} 3$ on common drugs used in transplantation. FMO3 SNP, rs1800822, was identified to be associated with tacrolimus troughs in non-African-American kidney transplant patients in a multicenter kidney transplant consortium study [21]. The influence of FMO3 genetic polymorphisms on the pharmacokinetic parameters of tacrolimus in Chinese liver transplant patients remains unclear.

In this study, we examined the $F M O 3$ genotypes ofliver transplant recipients and donors to clarify the influence of these genetic variants on tacrolimus dose requirements and pharmacokinetics post-transplantation.

\section{Patients \& methods}

\section{Study subjects}

In this retrospective observational study, 110 patients were enrolled who underwent liver transplantation at
Shanghai Jiao Tong University affiliated First People's Hospital from July 2007 to March 2012. The population, including 18 females and 92 males, were all Han Chinese. The average age was $47.5 \pm 8.8$ years and the average weight was $62.9 \pm 104.0 \mathrm{~kg}$. All patients received tacrolimus-based immunosuppressive regimens. The oral administration of tacrolimus was introduced in our previous study [7].

\section{Ethics statement}

This research was approved by the Ethics Committee of Shanghai Jiao Tong University. Informed written consent was obtained according to the Declaration of Helsinki.

\section{Data collection}

We used the tacrolimus serum concentration-to-dose ratios (C/D ratio) for 1 month post-transplantation as an index of tacrolimus pharmacokinetics as used in previous studies [7]. Trough tacrolimus blood concentration $(\mathrm{ng} / \mathrm{ml})$ was measured by PRO-TracTMII Tacrolimus ELISA kit (Diasorin, MN, USA) with microparticle enzyme immunoassay (ELx800NB analyzer, BioTek, USA). Tacrolimus daily dose (mg) was recorded and weight-adjusted dosage ( $\mathrm{mg} / \mathrm{kg}$ per day) was calculated. Tacrolimus C/D ratio was calculated by dividing tacrolimus trough concentration by the corresponding weight-adjusted dosage. Results of laboratory tests were also recorded. The average clinical data in different periods were calculated to represent the corresponding clinical status.

\section{Genotyping}

All the 27 FMO3 SNPs in the drug-metabolizing enzyme and transporter array, a standard platform for pharmacogenomics study, were selected for genotyped in this study. Both donor and recipient genomic DNA were extracted from liver tissues (stored at $-80^{\circ} \mathrm{C}$ ) using an AllPrep DNA/RNA Mini Kit (Qiagen, Hilden, Germany). SNPs were genotyped using Sequenom MassARRAY SNP-genotyping platform (Sequenom, CA, USA). The protocols included PCR amplification, shrimp alkaline phosphatase treatment, singlebase extension reaction, resin cleanup, nanodispensing on SpectroCHIP and data acquisition.

\section{Statistical analysis}

Chi-square test and Fisher's exact test were used to compare the distributions of genotypes and alleles of recipients and donors. Mann-Whitney $U$ and Kruskal-Wallis tests were used to assess the statistical significance of differences in C/D ratios for comparisons of all groups. Correlation between gene polymorphism and $\mathrm{C} / \mathrm{D}$ ratios was studied with univariate 
and multivariate linear regression analysis. Statistical analysis was performed using SPSS version 17.0 (SPSS Inc., IL, USA). Hardy-Weinberg equilibrium, allele frequency, linkage disequilibrium and haplotype analysis were analyzed using SHEsis software [22]. $\mathrm{p}<0.05$ was considered statistically significant. The Statistical graphs were made by Graphpad Prism version 5.00 software [23].

\section{Results}

\section{Gene polymorphisms}

The genotypes of the 27 FMO3 variants were shown in Supplementary Table 1. Rs2266782 (E158K), rs1736557 (V257M) and rs2266780 (E308G) that were previous found in various ethnic groups have a significant population distribution in the studied subjects. Rs1800822 (S147S) and rs909530 (N285N) that were rarely referred in other studies were found in the present study. Twenty-two SNPs were excluded for further analysis. Allele and genotype frequency of the remaining five SNPs and CYP3A5 rs776746 are shown in Table 1. There was no significance between donor and recipient genotype frequency $(\mathrm{p}>0.05)$. All six SNPs were in accordance with Hardy-Weinberg equilibrium ( $p>0.05)$. Rs2266782 and rs 2266780 were in strong linkage disequilibrium $\left(r^{2}=0.848\right.$; Figure 1).
Effect of single SNP on tacrolimus C/D ratios The effects of single-donor SNP on tacrolimus C/D ratios are shown in Table 2. Tacrolimus $\mathrm{C} / \mathrm{D}$ ratios of donors with $F M O 3$ SNP rs1800822 CC genotype were $131.9 \pm 123.9,115.6 \pm 97.8,128.3 \pm 93.8$ and $139.0 \pm 89.8$ at weeks $2,3,4$ and month 1 , respectively. For TC and TT genotype carriers, the corresponding tacrolimus $\mathrm{C} / \mathrm{D}$ ratios at each time point were $94.3 \pm 71.2,69.4 \pm 63.0,85.6 \pm 61.9$ and $99.0 \pm 82.5$. The differences were significant $(\mathrm{p}=0.016,0.002$, 0.026 and 0.008 , respectively). Donor FMO3 rs 909530 polymorphisms were associated with tacrolimus $\mathrm{C} / \mathrm{D}$ ratios at weeks 2,3 and month 1 ( $\mathrm{p}=0.001,0.003$ and 0.001 , respectively). Tacrolimus C/D ratios of donor rs909530 TC and TT carriers were very close and were significantly lower than those of CC genotype carriers. Tacrolimus C/D ratio of donor FMO3 rs2266780 AA genotype carriers was $131.4 \pm 117.6$ at week 2 , and GA and GG genotype carriers were $92.8 \pm 64.7$. The difference was significant $(\mathrm{p}=0.025)$. Tacrolimus $\mathrm{C} / \mathrm{D}$ ratios of donor CYP3A5 rs776746 GG genotype carriers were $278.3 \pm 396.9,130.8 \pm 103.2,119.3 \pm 79.1$, $144.7 \pm 123.2$ and $145.0 \pm 89.2$ at weeks $1,2,3,4$ and month 1 respectively. For AG and AA genotype carriers, the corresponding tacrolimus $\mathrm{C} / \mathrm{D}$ ratios were $176.0 \pm 197.8,102.4 \pm 101.3,82.8 \pm 89.3,83.0 \pm 73.0$ and $101.5 \pm 85.7$, respectively. The differences were

Table 1. Genotype and allele frequency of FMO3 and CYP3A5 polymorphism in liver transplant donors $(n=110)$ and recipients $(n=110)$.

\begin{tabular}{|c|c|c|c|c|c|c|}
\hline \multirow{2}{*}{$\begin{array}{l}\text { Gene } \\
\text { FMO3 }\end{array}$} & \multirow{2}{*}{$\begin{array}{l}\text { SNP } \\
\text { rs1800822 }\end{array}$} & \multicolumn{3}{|c|}{ Genotype frequency, \% (n) } & \multicolumn{2}{|c|}{ Allele frequency, \% (n) } \\
\hline & & $\mathrm{CC}$ & $\mathrm{TC}$ & TT & C & $\mathrm{T}$ \\
\hline & Donors & $0.69(76)$ & $0.29(32)$ & $0.02(2)$ & $0.84(184)$ & $0.16(36)$ \\
\hline & Recipients & $0.68(75)$ & $0.26(29)$ & $0.06(6)$ & $0.81(179)$ & $0.19(41)$ \\
\hline & rs2266782 & GG & AG & AA & G & $A$ \\
\hline & Donors & $0.63(69)$ & $0.30(33)$ & $0.07(8)$ & $0.78(171)$ & $0.22(49)$ \\
\hline & Recipients & $0.58(64)$ & $0.36(40)$ & $0.06(6)$ & $0.76(168)$ & $0.24(52)$ \\
\hline & rs1736557 & GG & AG & AA & G & $A$ \\
\hline & Donors & $0.63(69)$ & $0.33(36)$ & $0.04(5)$ & 0.79 (174) & $0.21(46)$ \\
\hline & Recipients & $0.66(73)$ & $0.31(34)$ & $0.03(3)$ & $0.82(180)$ & $0.18(40)$ \\
\hline & rs909530 & $\mathrm{CC}$ & $\mathrm{TC}$ & TT & $C$ & $\mathrm{~T}$ \\
\hline & Donors & $0.43(47)$ & $0.44(49)$ & 0.13 (14) & $0.65(143)$ & $0.35(77)$ \\
\hline & Recipients & $0.38(42)$ & $0.46(51)$ & $0.16(17)$ & $0.61(135)$ & $0.39(85)$ \\
\hline & rs2266780 & AA & GA & GG & A & G \\
\hline & Donors & $0.67(74)$ & $0.26(29)$ & $0.06(7)$ & $0.80(177)$ & $0.20(43)$ \\
\hline & Recipients & $0.60(66)$ & $0.34(38)$ & $0.06(6)$ & $0.77(170)$ & $0.23(50)$ \\
\hline \multirow[t]{3}{*}{ CYPЗА } & rs776746 & GG & AG & AA & G & A \\
\hline & Donors & $0.46(51)$ & $0.46(50)$ & $0.08(9)$ & 0.69 (152) & $0.31(68)$ \\
\hline & Recipients & $0.53(58)$ & $0.41(45)$ & $0.06(7)$ & $0.73(161)$ & $0.27(59)$ \\
\hline
\end{tabular}




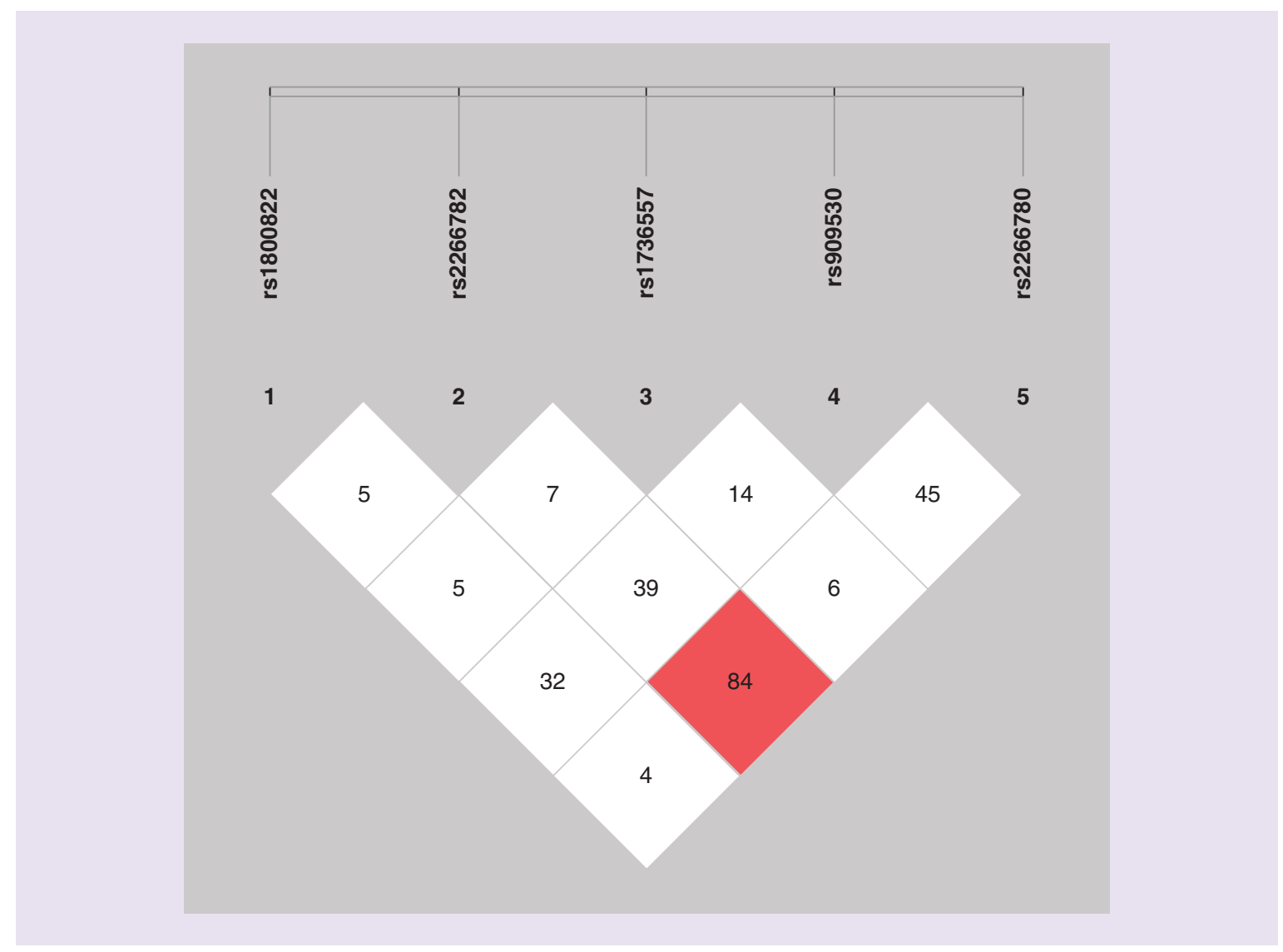

Figure 1. Linkage disequilibrium tests for five $F M O 3$ variants in our study subjects $(n=220)$.

significant at weeks $1,3,4$ and month 1 ( $\mathrm{p}=0.002$, $0.004,<0.001$ and 0.002 , respectively), and were nearly significant at week $2(\mathrm{p}=0.053)$.

The effects of single-recipient SNP on tacrolimus $\mathrm{C} / \mathrm{D}$ ratios are shown in Table 3 . The associations between tacrolimus $\mathrm{C} / \mathrm{D}$ ratios and recipient FMO3 gene polymorphisms were not significant except for rs1736557 polymorphisms at week $3(\mathrm{p}=0.030)$. Tacrolimus $\mathrm{C} / \mathrm{D}$ ratios of recipient $C Y P 3 A 5$ rs776746 GG genotype carriers were $285.4 \pm 265.6,132.8 \pm 104.6$, $116.1 \pm 99.6,130.7 \pm 97.0$ and $137.1 \pm 92.4$ at weeks $1,2,3,4$ and month 1, respectively, and 158.6 \pm 201.0, $100.6 \pm 93.5,86.8 \pm 84.3,90.4 \pm 81.9$ and $100.0 \pm 91.5$ for AG and AA genotype carriers. The differences were significant at every time point $(\mathrm{p}=0.002,0.029,0.010$, 0.013 and 0.007 , respectively).

Donor FMO3 rs1800822 and rs909530 were shown to be associated with tacrolimus elimination rate as stated above. Therefore, the two genotypes were further investigated in a combination analysis. Eight kinds of genotype combination were found in our study subjects. The percentages of $\mathrm{CC}+\mathrm{CC}$ (FMO3 rs1800822 CC and rs909530 CC), CC+TC (FMO3 rs1800822 CC and rs909530 TC), TC+TC (FMO3 rs1800822 TC and rs909530 TC) were 43, 20 and
$24 \%$. The three genotype combinations represent the overwhelming majority (87\%) of FMO3 genotype population distribution (Figure $2 \mathrm{~A}$ ). Tacrolimus $\mathrm{C} / \mathrm{D}$ ratios of donor $\mathrm{CC}+\mathrm{CC}, \mathrm{CC}+\mathrm{TC}$ and $\mathrm{TC}+\mathrm{TC}$ have significant differences in weeks $2,3,4$ and month 1 ( $\mathrm{p}=0.0022,0.0051,0.0466$ and 0.0221 , respectively; Figure 2C-F).

\section{Effects of FMO3 polymorphisms on tacrolimus C/D ratios in subjects with different CYP3A5 genotype backgrounds}

To eliminate the potential contribution of CYP3A5 polymorphisms on detected differences of tacrolimus $\mathrm{C} / \mathrm{D}$ ratios as results of FMO3 SNP types, we stratified patients by donor and recipient CYP3A5 expresser status and tested the effects of combined donor FMO3 rs1800822 and rs909530SNPs on tacrolimus C/D ratios in each group. As shown in Table 4, for both donor and recipient $C Y P 3 A 5^{*} 3 /^{*} 3$ (nonexpresser) groups, donor $F M O 3$ polymorphisms did not seem to have effect on tacrolimus $\mathrm{C} / \mathrm{D}$ ratios. The only significant differences were observed at week 2 in the donor nonexpresser group. On the other side, for both donor and recipient $C Y P 3 A 5^{*} 1$ (expresser) groups, the associations between tacrolimus $\mathrm{C} / \mathrm{D}$ ratios and donor 


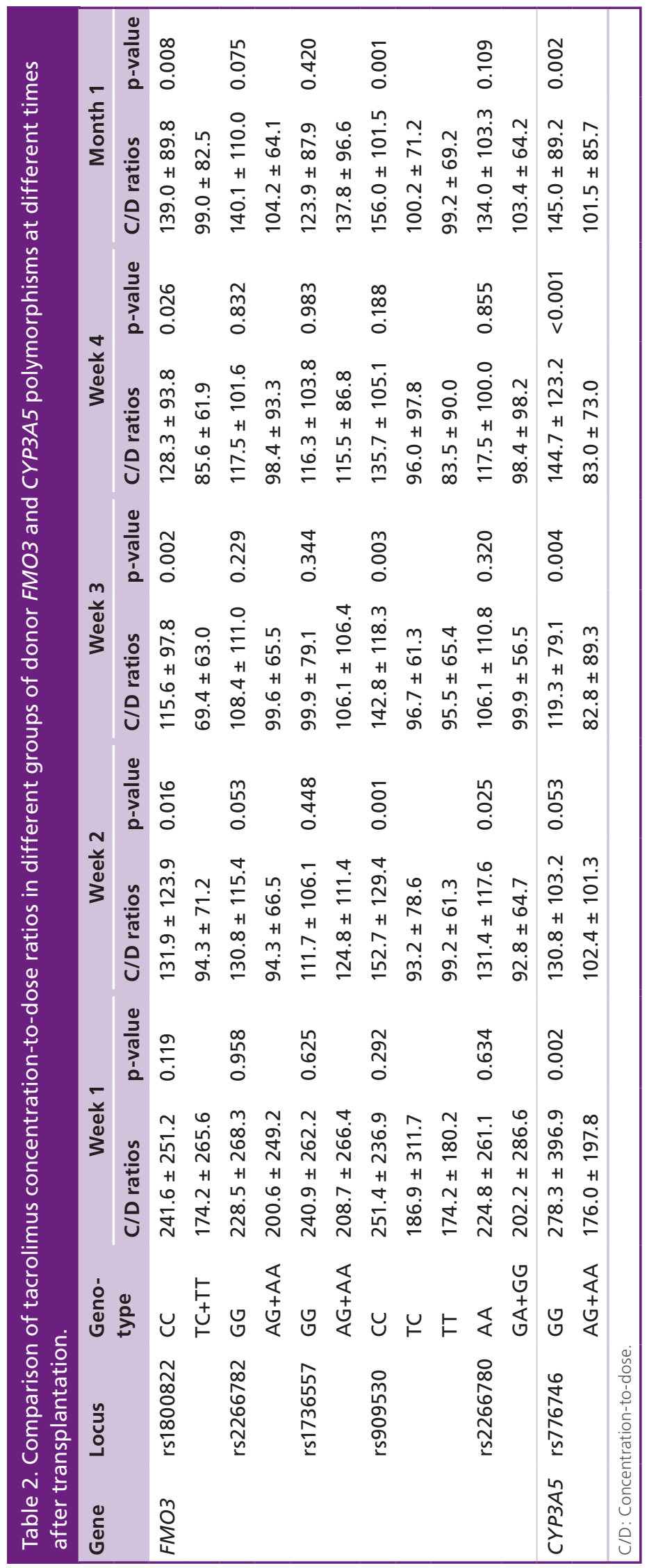




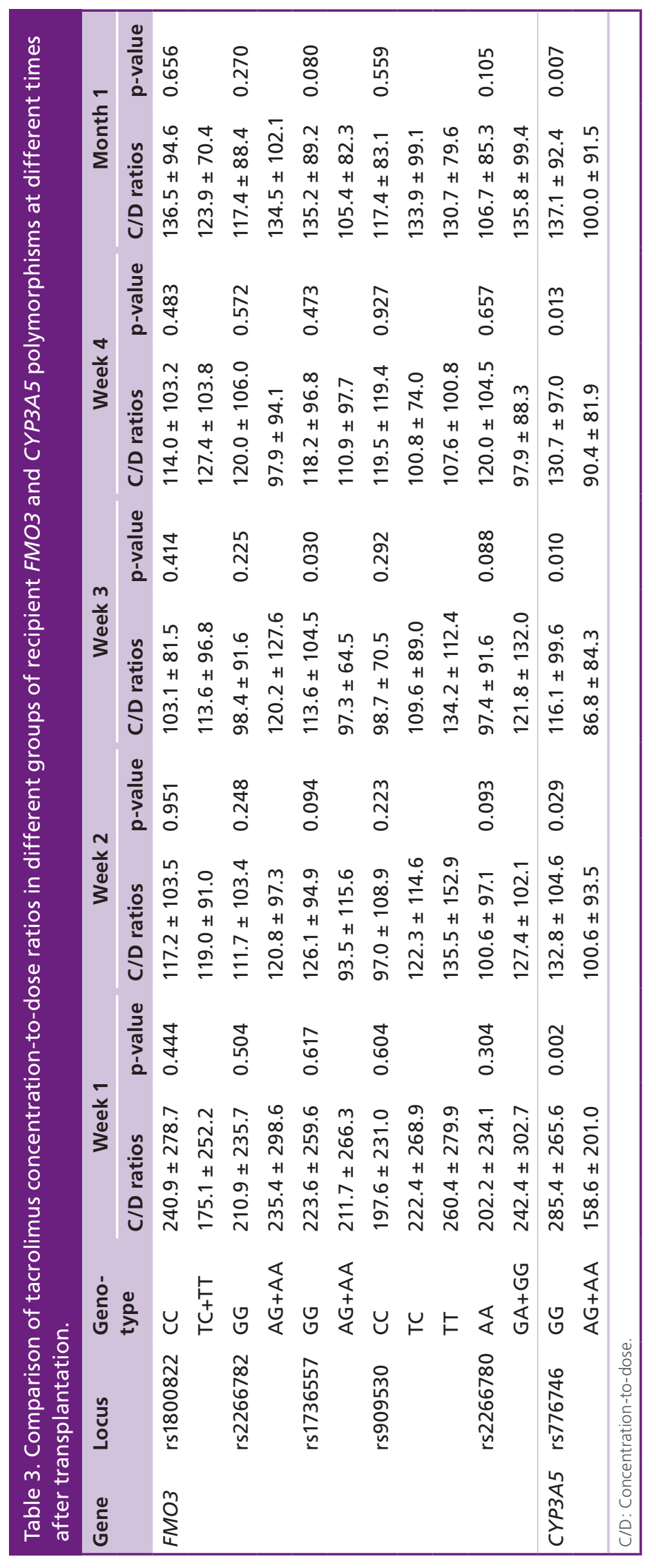

FMO3 gene polymorphisms were significant at every time point tested. These results indicate that although CYP3A5 is the major enzyme responsible for tacrolimus metabolism, in an rs776746 (expresser) genotype background when CYP3A5 expression is hindered, FMO3 might be a complementary pathway for tacrolimus metabolism. Therefore, the genotypes of FMO3 affect the pharmakinetics of tacrolimus.

\section{Multivariate analysis}

Multivariate linear regression analysis was used to screen the influence factors of tacrolimus pharmacokinetics. The incorporated cofactors included daily drug dose, donor and recipient CYP3A5 rs776746 genotypes, serum albumin concentration, red blood cell count, as well as liver function indexes (glutamate pyruvate transaminase, glutamicoxalacetic transaminase, total bilirubin, direct bilirubin), all reported to have the potential effects on tacrolimus pharmacokinetics, as well as the number of alleles associated with fast metabolism patients carrying in donor rs 1800822 and rs909530 sites. The results (Table 5) have shown that daily dose and donor and recipient CYP3A5 rs776746 genotypes are the consistent predictors except for week 2 . The number of donor rs 1800822 allele $\mathrm{T}$ and rs 909530 allele $\mathrm{T}$ was confirmed to be an independent predictor of C/D ratios for weeks 2 , 3 and 4.

\section{Discussion}

FMOs play an important role in the metabolism of a wide variety of foreign chemicals, including therapeutic drugs, dietary-derived compounds and pesticides [18]. However, in comparison with the CYPs, their role has largely been overlooked. Several reasons probably contribute to a lack of consideration of FMOs when the metabolism of a drug is investigated. FMOs are generally not inducible, they have no known specific inhibitors, the FMO proteins are thermally labile and it can be difficult to identify and quantify the products of FMO-catalyzed reactions [17,24]. FMO3 is a major FMOs enzyme in the human liver. Polymorphism of the FMO3 gene is known to influence the functionality of the FMO3 enzymes [18,25]. FMO3 rs1800822 was identified to be associated with tacrolimus troughs in non-African-American kidney transplant patients in a multicenter kidney transplant consortium study. In the present study, we have explored the association between $F M O 3$ polymorphisms and tacrolimus elimination in Chinese liver transplant patients.

Among 27 FMO3 SNPs analyzed, rs1800822 (S147S), rs2266782 (E158K), rs1736557 (V257M), rs909530 (N285N) and rs2266780 (E308G) variants have a relatively high population distribution in 
(A)

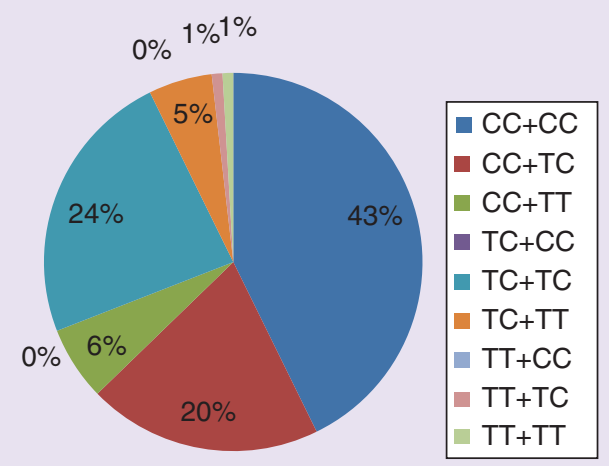

(c)

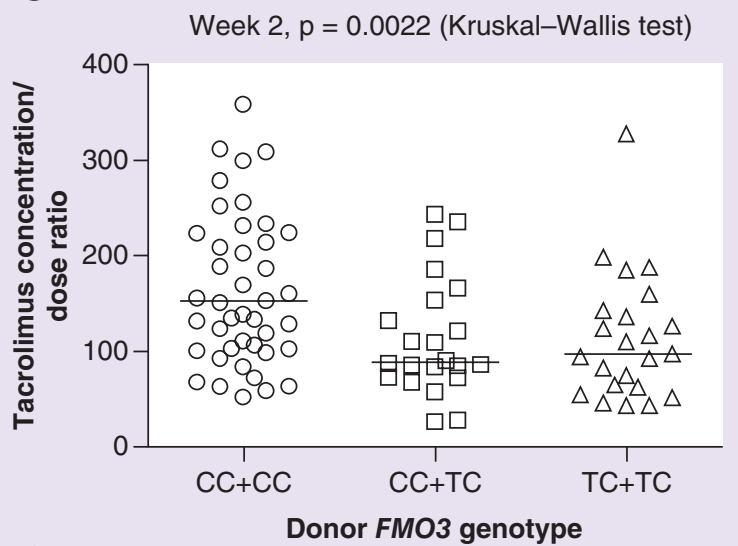

(E)

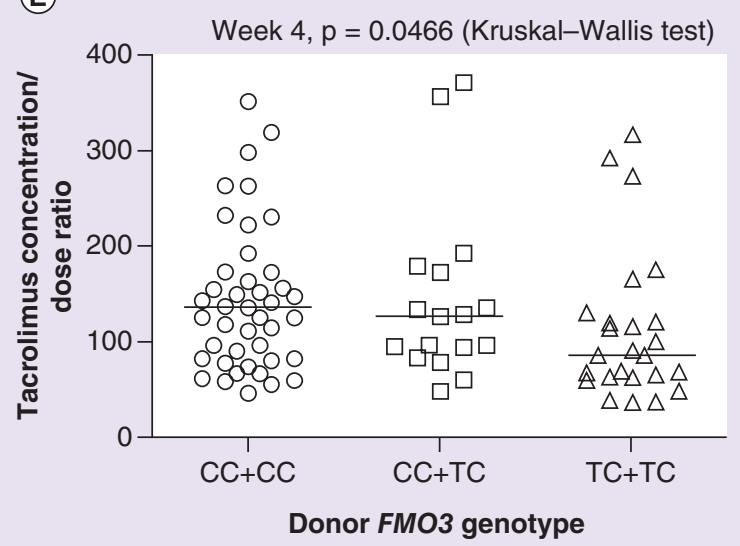

(B)

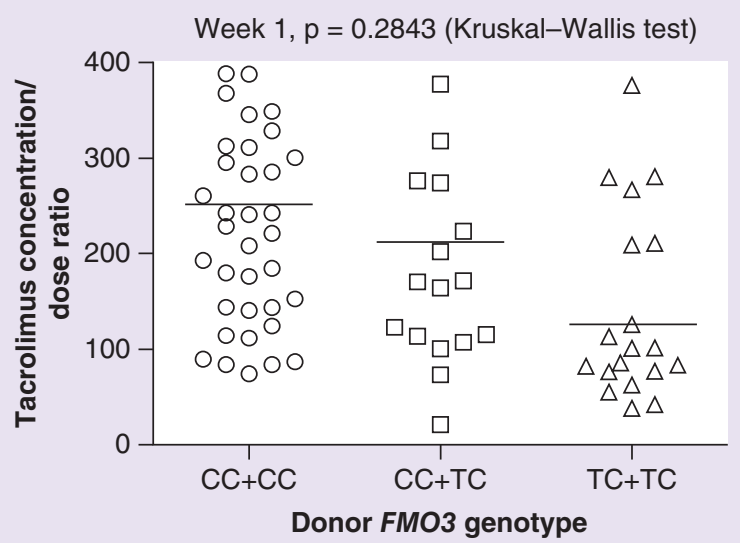

(D)
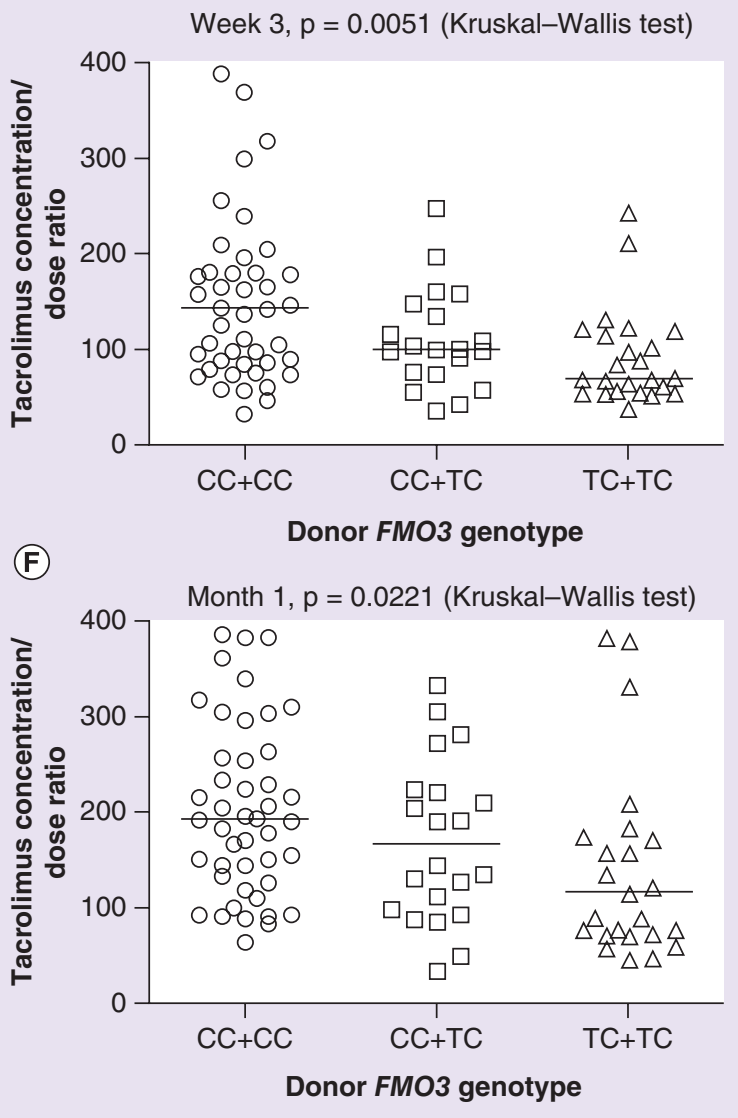

Figure 2. Combined analysis of donor FMO3 rs1800822 and rs909530 genotype on tacrolimus concentration-to-dose ratios. (A) FMO3 genotype population distribution, (B-F) The differences of tacrolimus C/D ratios of donor $C C+C C$, $\mathrm{CC}+\mathrm{TC}$ and TC+TC in weeks 1, 2, 3, 4 and month 1. CC+CC represents FM3 rs1800822 CC and rs909530 CC, CC+TC represents FMO3 rs1800822 CC and rs909530 TC, TC+TC represents FMO3 rs1800822 TC and rs909530 TC, then the rest $(\mathrm{CC}+\mathrm{TT}, \mathrm{TC}+\mathrm{CC}, \mathrm{TC}+\mathrm{TT}, \mathrm{TT}+\mathrm{CC}$ and $\mathrm{TT}+\mathrm{TC})$ and so on.

the studied subjects. No statistically significant differences in genotype and allele frequencies were observed between recipients and donors for any of the above genetic polymorphisms. E158K, V257M and E308G variants have been identified in all populations exam- ined so far including Caucasians, Asians and AfricanAmericans, with marked variability in frequency between major ethnicities [26,27]. In our data, the minor allele frequencies were 0.230 (E158K), 0.195 (V257M) and 0.211 (E308G). Cis-linkage between K158 and 


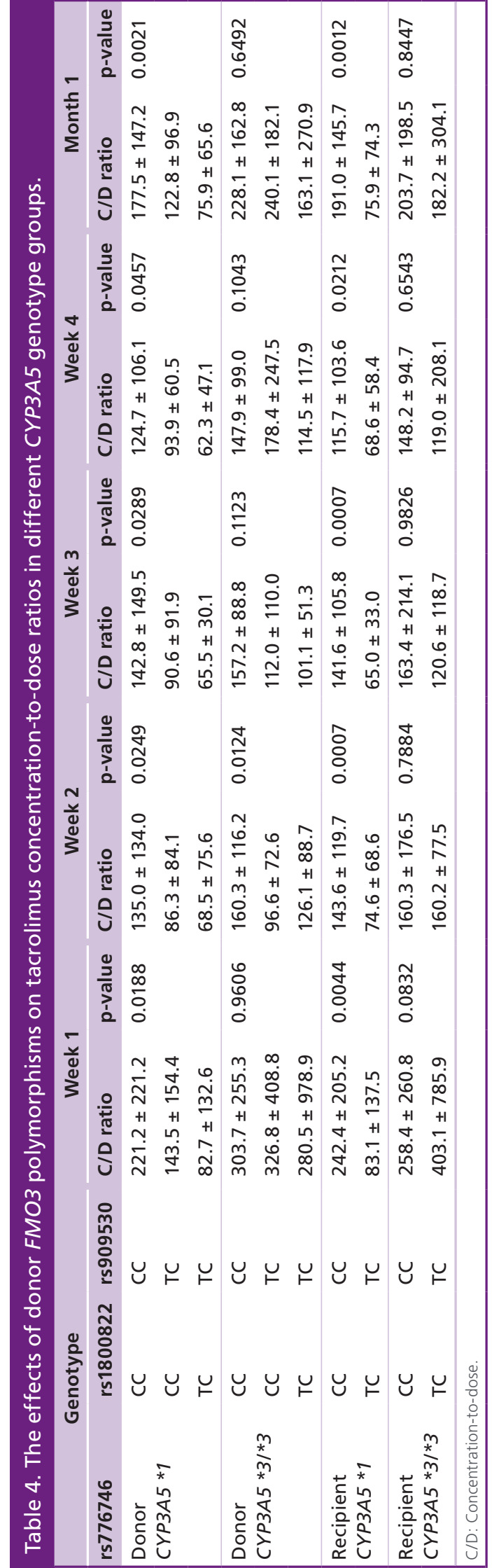

G308 has constantly appeared in all populations, with considerable variation in frequencies, of the inferred haplotype K158-G308 [28]. Among the five FMO3 polymorphisms, only sites 158 and 308 were also in significant linkage disequilibrium. The three variants can result in decreased FMO3 enzyme activity in a substrate-dependent manner. Trimethylaminuria is caused by a decreased ability of the FMO3 enzyme to oxidize trimethylamine. Previously, E158K, V257M and E308G inherited in particular combinations were shown to result in decreasing in trimethylamine oxidation and may predispose people to mild trimethylaminuria $[29,30]$. While E158K and E308G loci reduce activity in catabolizing sulindac, an NSAID, used as a chemo-preventive agent in patients with familial adenomatous polyposis, resulting in an increased efficacy to prevent polyposis in familial adenomatous polyposis [31,32]. However, our data did not show an association between tacrolimus elimination with E158K, V257M or E308G.

The other two synonymous exon variants, rs 1800822 and rs909530, were discovered by resequencing technology. Rs1800822 and rs909530 occurred at relatively high frequencies that differed among Hispanics (Mexican descent), African-Americans and nonLatino whites with significant ethnic differences [33]. In this study, we are the first to provide the allele and genotype frequency of the two FMO3 polymorphisms in the Han Chinese population. Rs909530 has been previously reported to be associated with toxicity due to docetaxel treatment including febrile neutropenia, reduced absolute neutrophil count and hemoglobin reduction [34]. The function of rs1800822 and rs909530 remains unclear. Rs1800822 and rs909530 were synonymous mutations, which are considered to be 'silent' as they do not affect the protein sequence. However, a 'silent' mutation may still affect protein expression through various mechanisms [35-37]. Our data showed that donor FMO3 rs1800822 allele T and rs909530 allele $\mathrm{T}$ were associated with fast tacrolimus limination. To our knowledge, the present study was the first analysis of the full extent of FMO3 genetic polymorphisms involving a drug-metabolizing enzyme and transporter array in a Han Chinese population.

The main metabolic organs of tacrolimus are the liver and intestine. A liver transplant patient is a good model for research on tacrolimus metabolism because the liver graft comes from the donor and intestine belongs to the recipient. In our data, donor but not recipient FMO3 polymorphisms were associated with tacrolimus elimination. This finding was consistent with FMO3 liver-specific expression in adults.

In our data, the pharmacokinetic influence of FMO3 polymorphisms was limited in the subjects 


\begin{tabular}{|c|c|c|c|c|c|c|}
\hline \multirow[t]{2}{*}{ Model } & & \multicolumn{2}{|c|}{ Unstandardized coefficients } & \multirow{2}{*}{$\begin{array}{l}\text { Standardized } \\
\text { coefficients } \\
\text { Beta }\end{array}$} & \multirow[t]{2}{*}{$\mathbf{t}$} & \multirow[t]{2}{*}{ p-value } \\
\hline & & B & Std. error & & & \\
\hline \multirow[t]{3}{*}{ Week 1} & (Constant) & 3.087 & 0.145 & & 21.302 & 0.000 \\
\hline & Donor rs776746 & -0.266 & 0.064 & -0.371 & -4.158 & 0.000 \\
\hline & Recipient rs776746 & -0.239 & 0.064 & -0.334 & -3.744 & 0.000 \\
\hline \multirow[t]{3}{*}{ Week 2} & (Constant) & 2.077 & 0.038 & & 54.115 & 0.000 \\
\hline & Total bilirubin & 0.002 & 0.000 & 0.368 & 4.294 & 0.000 \\
\hline & No. of $F M O 3$ alleles* & -0.083 & 0.022 & -0.323 & -3.772 & 0.000 \\
\hline \multirow[t]{4}{*}{ Week 3} & (Constant) & 2.597 & 0.115 & & 22.586 & 0.000 \\
\hline & No. of $F M O 3$ alleles* & -0.088 & 0.025 & -0.314 & -3.568 & 0.001 \\
\hline & Donor rs776746 & -0.165 & 0.051 & -0.287 & -3.252 & 0.002 \\
\hline & Recipient rs776746 & -0.138 & 0.051 & -0.241 & -2.734 & 0.007 \\
\hline \multirow[t]{5}{*}{ Week 4} & (Constant) & 2.563 & 0.114 & & 22.429 & 0.000 \\
\hline & No. of $F M O 3$ alleles* & -0.087 & 0.024 & -0.312 & -3.600 & 0.001 \\
\hline & Donor rs776746 & -0.176 & 0.050 & -0.307 & -3.513 & 0.001 \\
\hline & Recipient rs776746 & -0.137 & 0.050 & -0.239 & -2.756 & 0.007 \\
\hline & Total bilirubin & 0.002 & 0.001 & 0.179 & 2.058 & 0.042 \\
\hline
\end{tabular}

carrying $C Y P 3 A 5^{*} 1$ but not $C Y P 3 A 5^{*} 3 /^{*} 3$. This discrepancy come from the allele frequency of FMO3 rs1800822 and rs909530. The $\mathrm{T}$ allele frequencies of rs1800822, rs909530 were 16 and 35\%, respectively. FMO3 rs1800822 allele $\mathrm{T}$ and rs 909530 allele $\mathrm{T}$ were associated with fast tacrolimus elimination. The patients with fast tacrolimus elimination were prone to be screened using the genotype FMO3 rs1800822 and rs909530.

Many different factors were reported to have the potential effects on tacrolimus pharmacokinetics and may biase the results. The number of donor rs 1800822 allele $\mathrm{T}$ and rs 909530 allele $\mathrm{T}$ was confirmed to be an independent predictor of $\mathrm{C} / \mathrm{D}$ ratios for weeks 2,3 and 4 in the multivariate analysis.

Our study has several limitations. First, study population is relatively small and this limits the strength of conclusions. Due to the limited number of transplant operation, it is difficult to conduct the large sample pharmacogenomics research of tacrolimus. To our knowledge, the sample size of this study $(n=110)$ exceeded that of many pharmacogenomics researches of tacrolimus [7-10]. However, the association of FMO3 polymorphisms and tacrolimus elimination should be confirmed in further multicenter study with large sample size. Second, tacrolimus $\mathrm{C} / \mathrm{D}$ ratios beyond month 1 post-transplant (e.g., 6 and 12 months), in a more stable setting, were not observed in this study.
According to our clinical pracitce, individualized administration of tacrolimus in 1 month after liver transplantation had great impact on prognosis. We will concern the influence of FMO3 polymorphisms on tacrolimus $\mathrm{C} / \mathrm{D}$ ratios beyond month 1 post-transplant in further study.

\section{Conclusion \& future perspective}

In the present study, we conducted systematic genetic association analysis with tacrolimus elimination in Chinese liver transplant patients and first found that donor FMO3 rs1800822 allele $\mathrm{T}$ and rs 909530 allele $\mathrm{T}$ were associated with fast tacrolimus elimination. These results provided critical information for the development of personalized medicine with tacrolimus for Chinese liver transplant patients and may lead to optimal dosing recommendations reduced adverse drug reactions and improved drug efficacy.

\section{Supplementary data}

To view the supplementary data that accompany this paper please visit the journal website at: www.futuremedicine.com/ doi/full/10.2217/pgs-2016-0098

\section{Author contributions}

J Fan, L Ren, M Teng and T Zhang carried out the studies, participated in collecting data and drafted the manuscript. S Qin and J Fan performed the statistical analysis and participated in 
its design. B Sun and X Zhang helped to draft the manuscript. All authors read and approved the final manuscript.

Financial \& competing interests disclosure his work was supported by the National Natural Science Foundation of China (grant no. 81530044, 81202609, 81403010), Leading Project of Science and Technology Commission of Shanghai Municipality (134119a6300), Medical Engineering Crossing Project grant funded by Shanghai Jiaotong University (YG2012MS05) and the 863 Program (2012AA021002, 2012AA02A515, 2012AA021002). The authors have no other relevant affiliations or financial involvement with any organization or entity with a financial interest in or financial conflict with the subject matter or materials discussed in the manuscript apart from those disclosed.
No writing assistance was utilized in the production of this manuscript.

\section{Ethical conduct of research}

The authors state that they have obtained appropriate institutional review board approval or have followed the principles outlined in the Declaration of Helsinki for all human or animal experimental investigations. In addition, for investigations involving human subjects, informed consent has been obtained from the participants involved.

\section{Open access}

This work is licensed under the Attribution-NonCommercialNoDerivatives 4.0 Unported License. To view a copy of this license, visit http://creativecommons.org/licenses/by-nc-nd/4.0/

\section{Executive summary}

\section{Background}

- The FMO3 gene is very polymorphic and FMO3 variants have also been shown to be associated to many drug responses.

- FMO3 variants were potentially involved in tacrolimus metabolism in kidney transplantion. The influences of FMO3 genotypes on tacrolimus elimination in Chinese liver transplant patients remained unclear.

\section{Results}

- Donor FMO3 rs1800822 allele T and rs909530 allele T were associated with fast tacrolimus elimination.

- The number of donor rs1800822 allele T and rs909530 allele T was confirmed to be an independent predictor of the tacrolimus concentration-to-dose ratios for weeks 2, 3 and 4 in the multivariate analysis.

\section{References}

1 Starzl TE, Fung JJ. Themes of liver transplantation. Hepatology 51, 1869-1884 (2010).

2 Beckebaum S, Cicinnati VR, Radtke A, Kabar I. Calcineurin inhibitors in liver transplantation - still champions or threatened by serious competitors? Liver Int. 33, 656-665 (2013).

3 Arkas SA, Schnitzbauer AA, Kirchner G, Obed A, Banas B, Schlitt HJ. Calcineurin inhibitor minimization protocols in liver transplantation. Transpl. Int. 22, 49-60 (2009).

4 Wang L, McLeod HL, Weinshilboum RM. Genomics and drug response. N. Engl. J. Med. 364, 1144-1153 (2011).

5 Chen P, Lin JJ, Lu CS et al. Carbamazepine-induced toxic effects and $H L A-B^{*} 1502$ screening in Taiwan. N. Engl. J. Med. 364, 1126-1133 (2011).

6 Kurzawski M, Droździk M. Pharmacogenetics in solid organ transplantation: genes involved in mechanism of action and pharmacokinetics of immunosuppressive drugs. Pharmacogenomics 14, 1099-1118 (2013).

7 Zhang X, Wang Z, Fan J, Liu G, Peng Z. Impact of interleukin-10 gene polymorphisms on tacrolimus dosing requirements in Chinese liver transplant patients during the early posttransplantation period. Eur. J. Clin. Pharmacol. 67, 803-813 (2011).

8 Rojas LE, Herrero MJ, Bosó V et al. Meta-analysis and systematic review of the effect of the donor and recipient CYP3A5 6986A $>\mathrm{G}$ genotype on tacrolimus dose requirements in liver transplantation. Pharmacogenet. Genomics 23, 509-517 (2013).

9 Ji E, Choi L, Suh KS, Cho JY, Han N, Oh JM. Combinational effect of intestinal and hepatic CYP3A5 genotypes on tacrolimus pharmacokinetics in recipients of living donor liver transplantation. Transplantation 94, 866-872 (2012).

10 Yu S, Wu L, Jin J et al. Influence of CYP3A5 gene polymorphisms of donor rather than recipient to tacrolimus individual dose requirement in liver transplantation. Transplantation 81, 46-51 (2006).

11 de Jonge H, Kuypers DR. Pharmacogenetics in solid organ transplantation: current status and future directions. Transplant. Rev. (Orlando) 22, 6-20 (2008).

12 Elens L, Capron A, van Schaik RH et al. Impact of CYP3A4*22 allele on tacrolimus pharmacokinetics in early period after renal transplantation: toward updated genotype-based dosage guidelines. Ther. Drug Monit. 35, 608-616 (2013).

13 Yoon $\mathrm{SH}$, Cho JH, Kwon O et al. CYP3A and ABCBI genetic polymorphisms on the pharmacokinetics and pharmacodynamics of tacrolimus and its metabolites (M-I and M-III). Transplantation 95, 828-834 (2013).

14 Li D, Lu W, Zhu JY, Gao J, Lou YQ, Zhang GL. Population pharmacokinetics of tacrolimus and CYP3A5, MDR1 and $I L-10$ polymorphisms in adult liver transplant patients. J. Clin. Pharm. Ther. 32, 505-515 (2007).

15 Boivin AA, Cardinal H, Barama A et al. Influence of SLCO1B3 genetic variations on tacrolimus pharmacokinetics 
in renal transplant recipients. Drug Metab. Pharmacokinet. 28, 274-277 (2013).

16 Hines RN, McCarver DG. The ontogeny of human drug-metabolizing enzymes: Phase I oxidative enzymes. J. Pharmacol. Exp. Ther. 300, 355-360 (2002).

17 Shephard EA, Phillips IR. The potential of knockout mouse lines in defining the role of flavin-containing monooxygenases in drug metabolism. Expert Opin. Drug Metab. Toxicol. 6, 1083-1094 (2010).

Phillips IR, Shephard EA. Flavin-containing monooxygenases: mutations, disease and drug response. Trends Pharmacol. Sci. 29, 294-301 (2008).

19 Hines RN. Developmental and tissue-specific expression of human flavin-containing monooxygenases 1 and 3. Expert Opin. Drug Metab. Toxicol. 2, 41-49 (2006).

20 Hisamuddin IM, Yang VW. Genetic polymorphisms of human flavin-containing monooxygenase 3: implications for drug metabolism and clinical perspectives. Pharmacogenomics 8, 635-643 (2007).

21 Jacobson PA, Oetting WS, Brearley AM et al. Novel polymorphisms associated with tacrolimus trough concentrations: results from a multicenter kidney transplant consortium. Transplantation 91(3), 300-308 (2011).

22 SHEsis. http://analysis2.bio-x.cn/myAnalysis.php

23 GraphPad Software. www.graphpad.com

24 Krueger SK, Williams DE. Mammalian flavin-containing monooxygenases: structure/function, genetic polymorphisms and role in drug metabolism. Pharmacol. Ther. 106, 357-387 (2005).

25 Zhou J, Shephard EA. Mutation, polymorphism and perspectives for the future of human flavin-containing monooxygenase 3. Mutat. Res. 612, 165-171 (2006).

Hao D, Sun J, Furnes B et al. Allele and genotype frequencies of polymorphic FMO3 gene in two genetically distinct populations. Cell Biochem. Funct. 25, 443-453 (2007).

27 Hao DC, Sun J, Furnes B et al. Haplotype frequency distribution and linkage disequilibrium analysis of single nucleotide polymorphisms at the human $F M O 3$ gene locus. Biochem. Genet. 44, 391-407 (2006).
28 Mao M, Matimba A, Scordo MG et al. Flavin-containing monooxygenase 3 polymorphisms in 13 ethnic populations from Europe, east Asia and sub-Saharan Africa: frequency and linkage analysis. Pharmacogenomics 10, 1447-1455 (2009).

29 Yeung CK, Adman ET, Rettie AE. Functional characterization of genetic variants of human FMO3 associated with trimethylaminuria. Arch. Biochem. Biophys. 464, 251-259 (2007).

30 Lambert DM, Mamer OA, Akerman BR et al. In vivo variability of TMA oxidation is partially mediated by polymorphisms of the FMO3 gene. Mol. Genet. Metab. 73, 224-229 (2001).

31 Hisamuddin IM, Wehbi MA, Chao A et al. Genetic polymorphisms of human flavin monooxygenase 3 in sulindac-mediated primary chemoprevention of familial adenomatous polyposis. Clin. Cancer Res. 10, 8357-8362 (2004).

32 Hisamuddin IM, Wehbi MA, Schmotzer B et al. Genetic polymorphisms of flavinmonooxygenase 3 in sulindacinduced regression of colorectal adenomas in familial adenomatous polyposis. Cancer Epidemiol. Biomarkers Prev. 14, 2366-2369 (2005).

33 Koukouritaki SB, Poch MT, Henderson MC et al. Identification and functional analysis of common human flavin-containing monooxygenase 3 genetic variants. J. Pharmacol. Exp. Ther. 320, 266-273 (2007).

34 Awada Z, Haider S, Tfayli A et al. Pharmacogenomics variation in drug metabolizing enzymes and transporters in relation to docetaxel toxicity in Lebanese breast cancer patients: paving the way for OMICs in low and middle income countries. OMICS 17, 353-367 (2013).

35 Waldman YY, Tuller T, Keinan A, Ruppin E. Selection for translation efficiency on synonymous polymorphisms in recent human evolution. Genome Biol. Evol. 3, 749-761 (2011).

36 Okamoto K, Iwasaki N, Nishimura C et al. Identification of KCNJ15 as a susceptibility gene in Asian patients with Type 2 diabetes mellitus. Am. J. Hum. Genet. 86, 54-64 (2010).

37 Dunn PP, Hammond L, Coates E, Street J, Griner L, Darke C. "Silent" nucleotide substitution in exon 4 is responsible for the "alternative expression" of $H L A-A^{*} 01: 01: 38 \mathrm{~L}$ through aberrant splicing. Hum. Immunol. 72, 717-722 (2011). 